\title{
Cutaneous neonatal lupus: a transient under diagnosed entity
}

\begin{abstract}
In infants born to mothers with positive auto antibodies (Anti-Sjögren's-syndromerelated antigen A-SSA [also known as anti-Ro] and/or anti-Sjögren's syndrome type B-SSB [also called anti-La]), an active search of cutaneous lesions should be done. A biopsy may be necessary, and the result should be seen in the clinical setting. While the wounds heal without scarring, topical corticosteroid the rapy is required in some cases. Most patients with neonatal lupus have a transitory disease with spontaneous resolution within 4-6 months.
\end{abstract}

Keywords: neonates, cutaneous lupus, heart block
Volume 8 Issue 5 - 2018

\author{
Paulo Santos,' Cláudia Teles-Silva, ${ }^{2}$ Gustavo \\ Rocha ${ }^{2}$ Hercília Guimarães, 2,3 Maria João \\ Baptista, ${ }^{1,3}$ \\ 'Pediatric Cardiology Department, Centro Pediátrico Integrado, \\ Centro Hospital de São João, Porto, Portugal \\ ${ }^{2}$ Division of Neonatology, Centro Pediátrico Integrado, Centro \\ Hospital de São João, Porto, Portugal \\ ${ }^{3}$ Faculty of Medicine, Universidade do Porto, Portugal
}

\begin{abstract}
Correspondence: Éden Santos P, MD, Centro Hospitalar de São João, E.P.E. Alameda Professor, Hernâni Monteiro, 4200-319, Porto, Portugal, Tel +351910914664,

Email edenforever@live.com.pt
\end{abstract}

Received: July 23, 2018 | Published: September 10, 2018

\section{Case description}

A 3 days-old male patient born from a mother with positive auto antibodies, presented with erythematous scaly lesions, in the malar, per orbital and perinasal regions (Figure 1) (Figure 2), right lateral neck, chest, and abdomen these well-demarcated lesions appeared within a few hours after birth. Bradycardia was also noted and reverted spontaneously, and the 12-lead electro cardiogram was normal. Thee echocardiogram and hematological study were within normal values. His mother had positives anti-Sjögren's-syndrome-related antigen A and anti-Sjögren's syndrome type B. Due to his family history and physical findings the diagnosis of neonatal lupus syndrome was made. The histopathology analysis revealed degeneration of keratin ocytes and infiltration lymphocytes in the perivascular region; however, the immuno histochemical study was negative. Topic hydro cortisone acetate was started and, 4 days later no other complications were found and were discharge to home. In the follow up visit six months later there were no lesions observed. He suspended the treatment and was discharged from the outpatient consultation.

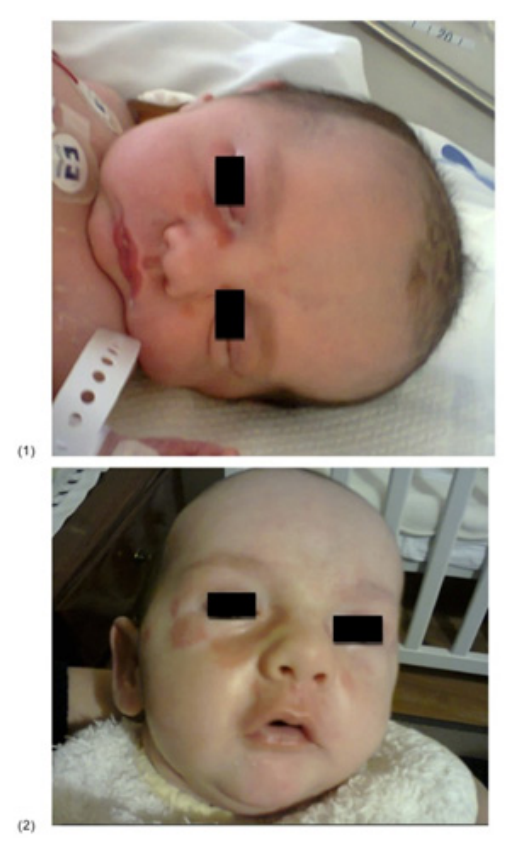

Figure I \& 2 


\section{Discussion}

Neonatal Lupus Syndrome is an auto immune disease associated with the presence of auto antibodies against ribonucleic proteins anti-Sjögren's-syndrome-related antigen A and/or anti-Sjögren's syndrome type B in the maternal-fetal circulation. Its feature may comprise congenital heart block, cutaneous and hematological manifestation. ${ }^{1,2}$ Cutaneous lesions are erythematous scaly plaques or annular polycyclic, photo sensitive, that resemble lupus lesions sub acute, distributed in regions most exposed to sunlight, mainly in the face (malar and periorbital regions), scalp and neck. Commonly, skin lesions appear few days or weeks after birth, and in many children, the injuries are initiated or exacerbated by exposure to sunlight. Due to its transient feature, the prevalence of cutaneous lesions may be underestimated in comparison to congenital heart block. ${ }^{3}$ Thus it's a transient expression with lasting approximately six months, coinciding with the disappearance of maternal G immuno globulin, in the infant's circulation. The histopathology is identical to subacute Lupus, ${ }^{1}$ albeit negative immuno histochemistry does not rule out the disease when there are suggestive features.

\section{Conclusion}

Neonatal lupus manifestations may include cutaneous involvement and/or congenital heart block. The former is common while the later is the mosts ever form of neonatal lupus. Anti-Ro and auto-anti bodies directed against cytoplasmic ribonucleic proteins are the main culprits responsible for this. These cross the placenta and cause damage to the immune system in the fetus. Congenital heart block is a serious consequence that may appear in utero and is permanent. The lesions heal spontaneously without marking, and topical corticosteroid therapy may be needed in some cases.

\section{Acknowledgements}

None.

\section{Compliance with ethical standards}

a. This study has no financial aid (no funding was received)

b. No conflict of interest

c. Thisreport does not contain any studies with human participants performed by any of the authors

d. Informed consent for patient in formation and images to be published was provided by a legally authorized representative.

\section{References}

1. Morel N, Georgin-Lavialle S, Levesque K, et al. Neonatal lupus syndrome: Literature review. Rev Med Interne. 2015;36(3):159-66.

2. Capone C, Buyon JP, Friedman DM, et al. Cardiac manifestations of neonatal lupus: a review of autoantibody-associated congenital heart block and its impact in an adult population. Cardiol Rev. 2012;20(2):72-76.

3. Yokogawa N, Sumitomo N, Miura M, et al. Neonatal lupus erythematosus. Nihon Rinsho Meneki Gakkai Kaishi. 2017;40(2):124-130. 